\title{
New Short Backfire Antennas for Wireless Applications
}

\author{
D. C. Thompson, R. L. Li, J. Papapolymerou, J. Laskar, and M. M. Tentzeris \\ School of Electrical and Computer Engineering \\ Georgia Institute of Technology, Atlanta, GA 30332-0250, USA
}

\begin{abstract}
A low-cost wideband short backfire antenna (SBA) and a circularly polarized (CP) SBA are presented. The low-cost SBA designed at the $5 \mathrm{GHz}$ UNII band is excited by a planar monopole which is printed on a dielectric substrate and fed by a microstrip line. By splitting the printed monopole with a slot, a wideband performance is achieved. It is demonstrated that the low-cost SBA can achieve an impedance bandwidth of $15 \%(\mathrm{VSWR}<2)$. The CP SBA is excited by a cross aperture with an unbalanced feed that consists of two orthogonal $\mathrm{H}$-shaped slots with a pair of capacitive stubs. It is shown that the CP SBA can achieve an axial ratio $(\leq 3 \mathrm{~dB})$ bandwidth of $4.2 \%$ with a VSWR bandwidth of $6.5 \%$ (VSWR $<1.2$ ) and a gain of $14 \mathrm{dBi}$. As highgain and low-cost wideband/CP directional antennas, the new SBAs can find applications in various wireless communications systems (such as LMDS, WLAN, and the emerging WiMAX networks) and wireless power transmission.
\end{abstract}

\section{INTRODUCTION}

In recent years, there has been an increasing need for high-gain wideband directional antennas in wireless applications [1], such as LMDS and WLAN. In particular, WiMax (world interoperability for microwave access), a technology based on an evolving standard for broadband point-to-multipoint wireless networking, is becoming a hot spot in the wireless industry [2]. The WiMax system needs fixed externally mounted directional subscriber antennas to communicate with base stations which are connected to the Internet. Since one of the major goals for wireless systems is to offer a less expensive infrastructure than a wired one, the cost-effectiveness of a wireless deployment is a big issue of concern. For some wireless applications, such as wireless power transmission [3], it is also desirable to employ a circularly polarized (CP) antenna to avoid the power loss caused by an unpredicted rotation of receive and/or transmit antennas.

The short backfire antenna (SBA) developed first in the 1960s [4] may become one of the most competitive candidates for these wireless application because of its high gain and simple structure. The most popular excitation for the SBA is a simple dipole antenna. Unfortunately the dipole-excited SBA has a narrow bandwidth for its input impedance [5]. For a CP SBA, the commonly used excitation is a cross dipole that consists of two orthogonal dipoles. Usually the cross dipole requires a balun circuit and a $90^{\circ}$ hybrid coupler for CP radiation. In this paper, two new SBAs are proposed to enhance the impedance bandwidth and to achieve $\mathrm{CP}$ radiation with a single feed. The wideband SBA is excited by a planar monopole fed by a microstrip line. Both the monopole and the microstrip line are printed on the same dielectric substrate, which is helpful in lowering the manufacturing cost by taking advantage of modern printed circuit technologies. By splitting the printed monopole with a slot, a wideband performance can be achieved. The CP SBA is excited by a cross aperture with an unbalanced feed consisting of two orthogonal H-shaped slots. The unbalanced feed is formed by a feed probe with a shorting pin. A pair of short stubs is introduced in the cross aperture to enhance the capacitive component of the longer H-shaped slot, allowing a decrease in the size of the cross aperture.

\section{A LOW-COST WIDEBAND SBA}


The geometry of the low-cost wideband SBA designed at a center frequency of $f_{c}=5.5$ $\mathrm{GHz}$ is depicted in Fig. 1. The SBA contains a primary reflector, a sub-reflector, and a circular rim. The excitation structure consists of a planar monopole and a microstrip line, both of which are printed on the same dielectric substrate (RT/duroid 5880, $\varepsilon_{\mathrm{r}}=2.2$ and thickness $=0.508 \mathrm{~mm}$ ). To achieve a wideband performance for the input impedance, the printed monopole is split along its central line into two parts with a slot. The split monopole is mounted at the center of the primary reflector and is fed across the splitting slot by a feed probe which is formed through an extension of a $50-\Omega$ microstrip line. All the geometric parameters are optimized by simulations. The overall dimensions of optimized SBA are $0.64 \lambda_{0}\left(\lambda_{0}=\right.$ the free-space wavelength at $\left.5.5 \mathrm{GHz}\right)$ in height and $2.2 \lambda_{0}$ in diameter.

Fig. 2 shows the simulated and measured results of VSWR for the new SBA and good agreement is observed. The VSWR $<2$ bandwidth (about $15 \%$ ) covers entirely the $5 \mathrm{GHz}$ USII band (5.1-5.9 GHz). The simulated directivity and measured power gain are plotted in Fig. 3. It can be seen that the power gain, slightly lower than the directivity, keeps near $15 \mathrm{dBi}$ over the $5 \mathrm{GHz}$ USII bands. The simulation reveals that the radiation efficiency of the SBA is higher than $95 \%$ over the $5 \mathrm{GHz}$ USII band. The simulated and measured results for the half-power beam width (HPBW) also agree well. The HPBW in the Eplane maintains almost a constant of $35^{\circ}$ while the HPBW in H-plane decreases as the frequency increases. At the center frequency $(5.5 \mathrm{GHz})$ the HPBW in the E-plane is about $10^{\circ}$ wider than that in the H-plane. The aperture efficiency at the center frequency is about $\eta_{\mathrm{a}}=70 \%$.

\section{A CROSS-APERTURE EXCITED CP SBA}

The configuration of the cross-aperture excited CP SBA designed for $5.8 \mathrm{GHz}$ ISM band $(5.7-5.9 \mathrm{GHz})$ is illustrated in Fig. 4. The excitation structure consists of a cross aperture fed by a single probe with a shorting pin. The cross aperture is formed by two orthogonal $\mathrm{H}$-shaped slots (Slots X and Y) etched on a circular patch whose diameter is equal to that of the sub-reflector. The H-shaped slot is divided into a wider part and a narrower part. The purpose of the wider part is to increase the effective length of each slot, hence making the slot close to resonance. The reason for introducing a narrower part in the slot is to excite over the cross aperture a stronger electric-field distribution which is coupled to the reflectors of the SBA. In order to accomplish a phase difference between the radiated fields from the two slots, Slot $\mathrm{X}$ is set to be $0.1 \lambda_{0}$ longer than Slot $\mathrm{Y}$. This length difference is not enough for a $90^{\circ}$ phase difference required by a CP antenna. Therefore a pair of short stubs connecting respectively to the feed probe and the shorting pin is added to the middle of Slot X. The pair of short stubs can be thought of as a capacitor parallel to Slot $\mathrm{X}$, making the slot more capacitive and increasing the effective length. The overall dimensions of the CP SBA are $0.54 \lambda_{0}\left(\lambda_{0}=\right.$ the free-space wavelength at $\left.5.8 \mathrm{GHz}\right)$ in height and $1.93 \lambda_{0}$ in diameter. The diameter and height of the slotted patch are $0.46 \lambda_{0}$ and $0.27 \lambda_{0}$, respectively. The width of the wider part of the H-shaped slots is chosen to be 6 times the width of the narrow part that is optimized to be $1.0 \mathrm{~mm}$. The total lengths of Slots $\mathrm{X}$ and $\mathrm{Y}$ are respectively $18 \mathrm{~mm}\left(\sim 0.35 \lambda_{0}\right)$ and $13 \mathrm{~mm}\left(\sim 0.25 \lambda_{0}\right)$. The length and the width of the capacitive stubs are adjusted to $2.5 \mathrm{~mm}$ and $0.3 \mathrm{~mm}$. The diameter and the center-to-center distance of the feed probe and the shorting pins are $2 \mathrm{~mm}$ and 4.2 $\mathrm{mm}$, respectively.

Fig. 5 shows the simulated and measured results for VSWR of the CP SBA. Good agreement is obtained. The bandwidth for VSWR $<1.2$ is found to be about $6.5 \%$, much better than the conventional CP SBA [5]. The axial ratio (AR) at broadside and gain of 
the new CP SBA are plotted in Fig. 6. It is seen that the bandwidth for $\mathrm{AR} \leq 3 \mathrm{~dB}$ is about $4.2 \%$ and the gain is around $14 \mathrm{dBi}$. The aperture efficiency is near $80 \%$. The radiation patterns of the wideband SBA and the CP SBA will be presented at the conference.

\section{CONCLUSION}

A low-cost wideband SBA and a CP SBA are developed for wireless applications. The low cost SBA is excited by a planar monopole which is printed on the same dielectric substrate with its feeding microstrip line. This configuration is helpful in reducing the manufacturing cost. The impedance bandwidth of the new SBA is found to be about $15 \%$ for VSWR $<2$. The CP SBA is excited by a cross aperture that consists of two orthogonal $\mathrm{H}$-shaped slots fed by a single probe with a shorting pin. A pair of capacitive stubs is introduced to reduce the size of the cross aperture. The new CP SBA has an excellent input impedance matching (a bandwidth of $6.5 \%$ for VSWR $<1.2$ ) and the advantage of not requiring a balun circuit or an external polarizer. It is shown that the cross-aperture excited SBA achieves an axial ratio $(\leq 3 \mathrm{~dB})$ bandwidth of $4.2 \%$ with a gain of $14 \mathrm{dBi}$. The new SBAs may find applications in broadband wireless communications (such as LMDS, WLAN, and WiMax) and wireless power transmission.

\section{Acknowledgement}

The authors wish to acknowledge the support of Georgia Electronic Design Center, the NSF CAREER Award under contract NSF \#9964761, the NSF Award NSF ECS-0313951, and the NSF Packaging Research Center,

\section{References}

[1] P. F. Driessen, "Gigabit/s indoor wireless systems with directional antennas," IEEE Trans. On Communications, vol. 44, no. 8, pp. 1034-1044, Aug. 1996.

[2] S. J. Vaughan-Nichols, "Achieving wireless broadband with MiMax," Computer, pp. 10-13, June 2004.

[3] J. Heikkinen and M. Kivikoski, "Low-profile circularly polarized rectifying antenna for wireless power transmission at $5.8 \mathrm{GHz}$," IEEE Microwave and Wireless Components Lett., vol. 14, no. 4. pp. 162-164, 2004

[4] H. W. Ehrenspeck, "The short-backfire antenna," Proc. IEEE. vol. 53, no. 6, pp. 1138-1140, Aug. 1965.

[5] S. Ohmori, S. Miura, K. Kameyama, and H. Yoshimura, "An improvement in electrical characteristics of a short backfire antenna," IEEE Trans. Antenna and Propagat., vol. 31, no. 4, pp. 644-646, July 1983.

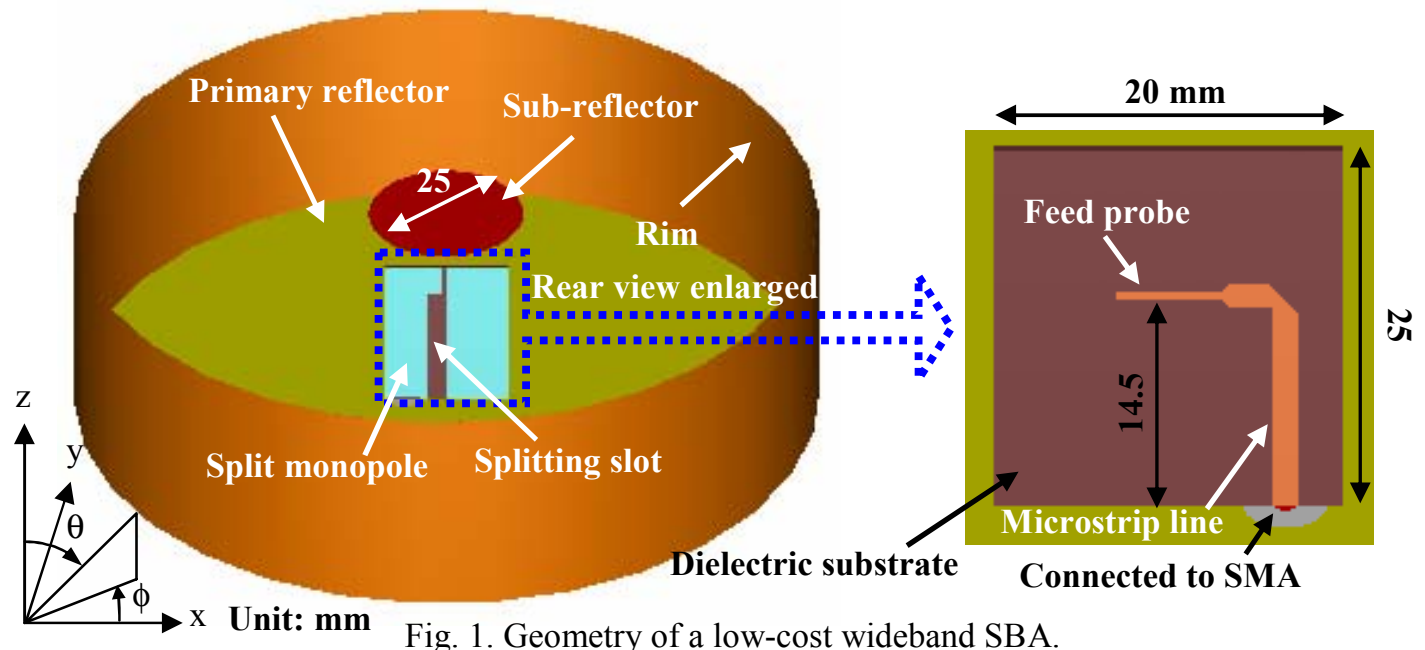

Fig. 1. Geometry of a low-cost wideband SBA. 


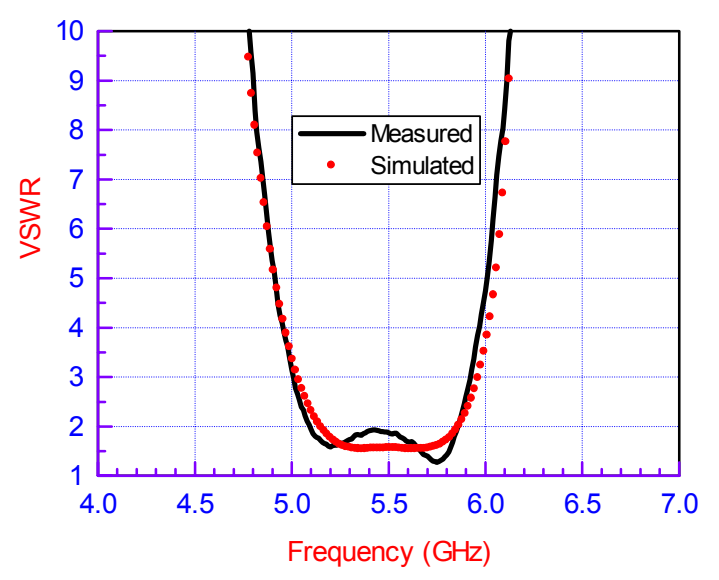

Fig. 2. Measured and simulated results of VSWR for the low-cost wideband SBA.

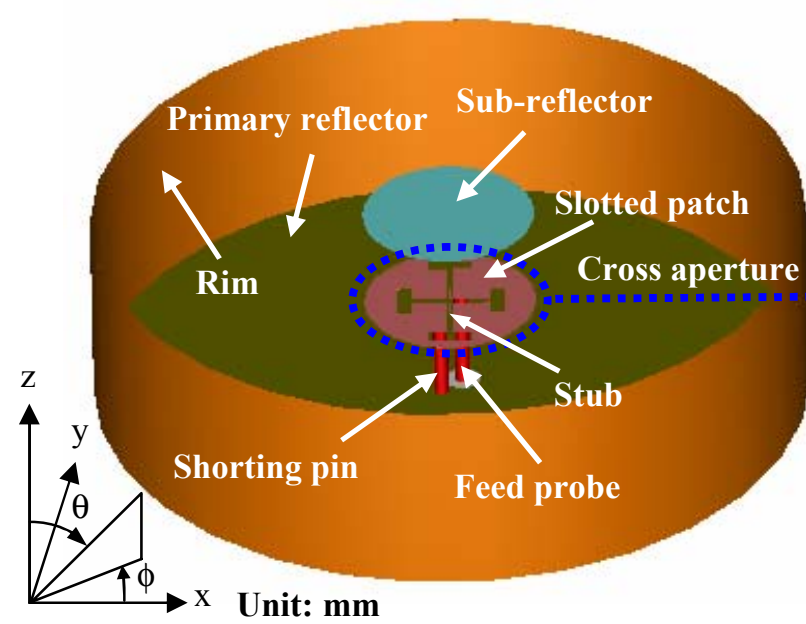

Fig. 4. Configuration of a cross-aperture excited CP SBA.

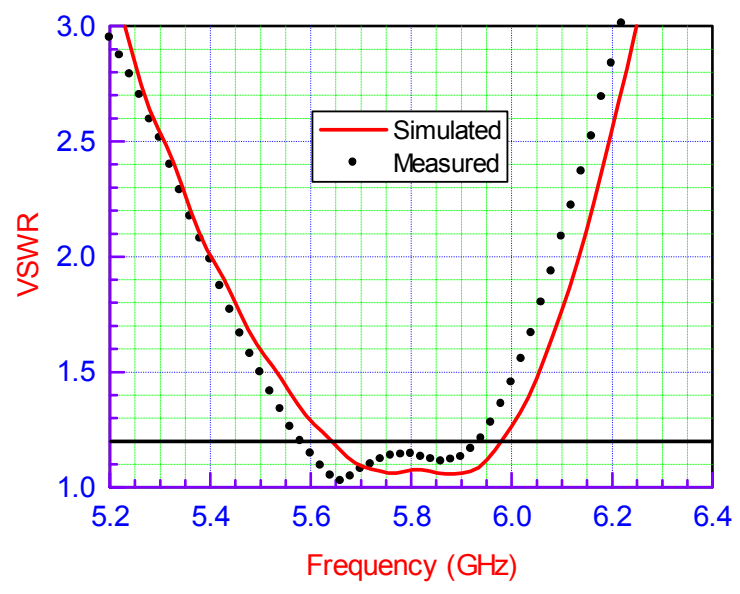

Fig. 5. Measured and simulated results of VSWR for the cross-aperture excited SBA. cross-aperture excited SBA.

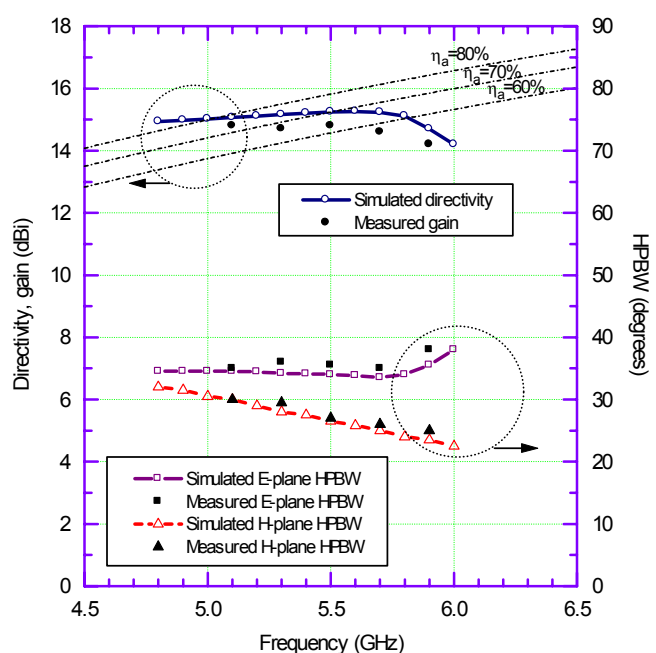

Fig. 3. Directivity, gain, and halfpower beam width (HPBW) of the low-cost wideband SBA.

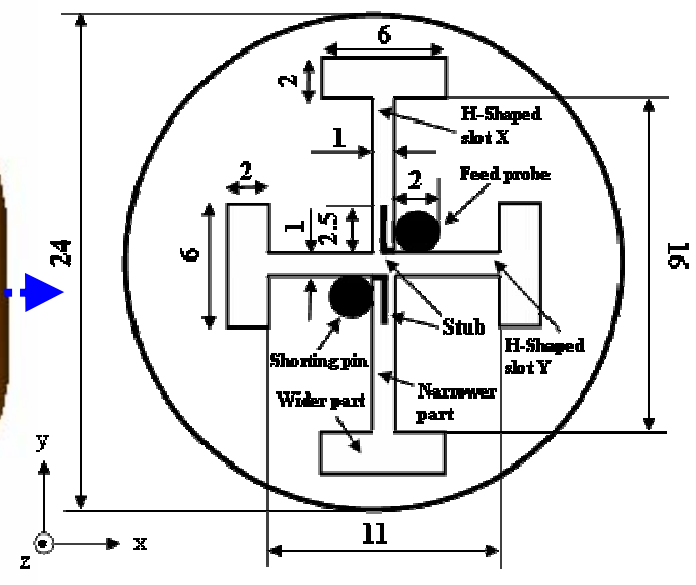

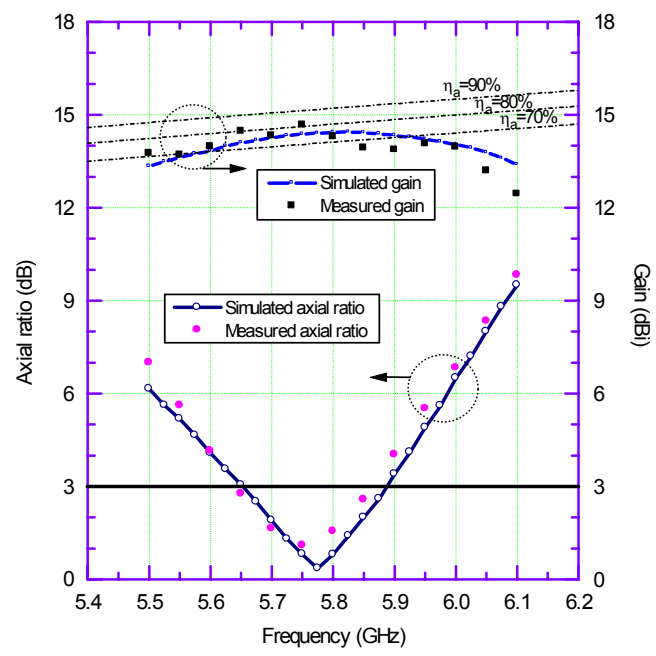

Fig. 6. Axial ratio and gain of the 